\title{
Prediction of hamstring injury in professional soccer players by isokinetic measurements
}

\author{
Marc Dauty ${ }^{1,2}$ \\ Pierre Menu ${ }^{1}$ \\ Alban Fouasson-Chailloux ${ }^{1}$ \\ Sophie Ferréol ${ }^{1}$ \\ Charles Dubois ${ }^{1}$ \\ 1 Department of Pôle MPR, Hôpital Saint Jacques, \\ CHU NANTES, France \\ 2 INSERM, UMRS 791-LIOAD, Centre for osteo- \\ articular and dental tissue engineering, groupe \\ STEP, Nantes, France
}

Corresponding author:

Marc Dauty

Department of Pôle MPR, Hôpital Saint Jacques, CHU NANTES

85 Rue Saint Jacques

44035 Nantes, France

E-mail: marc.dauty@chu-nantes.fr

\section{Summary}

Objectives: previous studies investigating the ability of isokinetic strength ratios to predict hamstring injuries in soccer players have reported conflicting results.

Hypothesis: to determine if isokinetic ratios are able to predict hamstring injury occurring during the season in professional soccer players.

Study Design: case-control study; Level of evidence: 3.

Methods: from 2001 to 2011,350 isokinetic tests were performed in 136 professional soccer players at the beginning of the soccer season. Fifty-seven players suffered hamstring injury during the season that followed the isokinetic tests. These players were compared with the 79 uninjured players. The bilateral concentric ratio (hamstring-to-hamstring), ipsilateral concentric ratio (hamstring-toquadriceps), and mixed ratio (eccentric/concentric hamstring-to-quadriceps) were studied. The predictive ability of each ratio was established based on the likelihood ratio and post-test probability.

Results: the mixed ratio (30 eccentric/240 concentric hamstring-to-quadriceps) $<0.8$, ipsilateral ratio (180 concentric hamstring-to-quadriceps) $<0.47$, and bilateral ratio ( 60 concentric hamstring-to-hamstring) $<0.85$ were the most predictive of hamstring injury. The ipsilateral ratio $<0.47$ allowed prediction of the severity of the hamstring injury, and was also influenced by the length of time since administration of the isokinetic tests.

Conclusion: isokinetic ratios are useful for predicting the likelihood of hamstring injury in professional soccer players during the competitive season.

KEY WORDS: eccentric, muscle contraction, sports, strength.

\section{Introduction}

The practice of soccer requires force, speed, concentration as well as precision ${ }^{1}$. Trainings and matches intensity is the cause of numerous muscle injuries which occurs particularly at the hamstrings muscle and most commonly on the biceps femoris muscle ${ }^{2}$. The incidence of hamstring injury is estimated of $15 \%$ in one season with a rate of recurrence of $30 \% 1,3$. The management of muscle injuries depends from the clinical diagnosis ${ }^{4}$. After hamstring strain injuries, clinical factors as pain scale and pain during everyday activities can predict the time to return to competition $^{5}$. The prevention of the muscular injuries seems multifactorial and would imply nutrition and hydration to optimize performances and recovery, type of grounds, climatic conditions, or still stretching and strengthening protocols to restore limbs muscle imbalance ${ }^{6,7}$. Since systematic isokinetic strength evaluations at the high-level sportsmen, a causal link between muscle imbalance and the occurrence of thigh muscle injuries is suspected ${ }^{8}$. The mixed or functional ipsilateral ratio (eccentric/concentric hamstring-toquadriceps) would correspond to the most interesting ratio to identify a previous muscle injury ${ }^{9,10}$. Furthermore, several Authors recommend rehabilitation at players who present a muscle imbalance to prevent a new muscle injury ${ }^{9,11-13}$. However, if the mixed ratios can allow the revealing of a previous muscle injury, it is not proved that these ratios can predict a new muscle injury. In our knowledge, only two studies measured the prediction of isokinetic strength tests for this pathology 8,14 . According to the cut-off values of isokinetic ratios which one chosen, the results were contradictory. Zvizac et al. did not show a predictive interest of the conventional concentric ratios at the American football player if the cut-off value of 0.6 was retained ${ }^{14}$. On the contrary, Croisier et al. showed a decrease of the number of hamstring muscle injuries 
during the correction of the mixed (eccentric/concentric) ratio inferior of $0.8^{8}$. From this report, our study compared the various isokinetic strength ratios at professional soccer players according to the occurrence or not of muscle hamstring injury during the season which followed isokinetic tests. In a second time, the capacity of isokinetic strength ratios of the quadriceps and hamstrings to predict the occurrence of a new muscle injury was calculated. This prediction was also studied according to the severity of injury, moderate or major, and according to the period between isokinetic tests and the occurrence of the new muscle injury ${ }^{15}$.

\section{Materials and methods}

\section{Population}

All the players who practised professional soccer in a French league 1 club between the season 2001-02 and 2011-12 were listed from the official Internet site of the club without perceiving financial advantage. Ethics approval was obtained from the internal review board of the French Football Club of Nantes and the study was performed in accordance with the ethical standards in Sports Medicine ${ }^{16}$. An informed consent was signed by each soccer player at the beginning of the different sport seasons. To ensure team and player confidentiality, all performance data were anonymized before analysis.

Only the male players, who were able to play in competition at least 20 matches during the professional soccer season were included if they were evaluated by isokinetic strength tests at the season beginning. The young players of the training centre and the professional players who presented significant injury of the lower limbs in the year which preceded isokinetic strength tests were excluded. The knee surgeries, quadriceps injuries, and hamstring injuries having obliged to stop training of more than 7 days, were excluded because of the durable loss of force that these various pathologies can engender ${ }^{10,17}$.

\section{Parameters of study}

The age, weight, height and the individual playing positions were considered (goalkeeper, defender, midfield and forward). Only the intrinsic hamstring muscle injuries were identified from the professional sport injury register over the period 2001 to 2012. All muscle injuries were clinically diagnosed and confirmed by ultrasound. The minor muscle injuries (less than 7 days of sport stop) were excluded because of the risk to include a not muscle pathology or to minimized the incidence of these minor injuries by absence of declaration in the professional sport injury register ${ }^{15}$. The right or left side, the severity of injury ( $\leq 28$ days or $>$ 28 days) and the period between isokinetic test and the occurrence of injury ( $\leq 1$ month or between 1 and 3 months or $>3$ months) were specified ${ }^{18}$.

\section{Strength Measurements}

Isokinetic strength tests were performed using a Cybex Norm ${ }^{\circledR}$ dynamometer (Lumex Inc. Ronkoma, NY, USA) at the beginning of season (June, July, August). After controlled 10 minutes cycloergometer (Technogym $^{\circledR}$, Gambettola, Italy) warm up (100 watts at 70 $\mathrm{rpm}$ ), the subject sat with the thigh at an angle of 85 degrees to the trunk, measured by manual goniometry. The mechanical axis of the dynamometer was aligned with the knee lateral epicondyle. The knee range of motion was 100 degrees ( 0 degrees of extension to 100 degrees of flexion). Torque was gravity-corrected and the dynamometer calibration as performed in accordance with the manufacturer instructions. An adequate familiarization with the dynamometer was provided in the form of 3 submaximal followed by 2 maximal concentric contractions at 60 degrees. The two sides were evaluated in a random order. All subjects benefited from verbal encouragements and a visual feedback.

During 10 years of study, three isokinetic protocols were used. They consisted of 4 series among which 2 according to concentric muscle contractions and 2 according to eccentric muscle contractions spaced out of 30 seconds rest (Tab. 1).

\section{Isokinetic parameters}

The knee flexors and extensors maximal peak torques according to different angular speed and muscle contraction modes were measured, in Newton meter, to calculate the various strength ratios (Tab. 2) ${ }^{19-21}$. Moderate relative reliability was established for strength ratio with eccentric hamstring-to-concentric quadriceps ratio showing the greater intraclass correlation coefficient $(0.87)^{18}$.

Table 1. Various isokinetic protocols used during 10 soccer seasons.

\begin{tabular}{lll}
\hline Protocol 1 & Protocol 2 & Protocol 3 \\
\hline Con $60 \mathrm{deg} / \mathrm{s} \times 3$ & Con $60 \mathrm{deg} / \mathrm{s} \times 3$ & Con $120 \mathrm{deg} / \mathrm{s} \times 3$ \\
Con $180 \mathrm{deg} / \mathrm{s} \times 5$ & Con $240 \mathrm{deg} / \mathrm{s} \times 5$ & Con $240 \mathrm{deg} / \mathrm{s} \times 5$ \\
Ecc $60 \mathrm{deg} / \mathrm{s} \times 5$ & Ecc $30 \mathrm{deg} / \mathrm{s} \times 5$ & Ecc $30 \mathrm{deg} / \mathrm{s} \times 5$ \\
Ecc $120 \mathrm{deg} / \mathrm{s} \times 5$ & Ecc $120 \mathrm{deg} / \mathrm{s} \times 5$ & Ecc $120 \mathrm{deg} / \mathrm{s} \times 5$ \\
\hline
\end{tabular}

Abbreviations: Con: concentric; Ecc: eccentric. 
Table 2. Calculated isokinetic strength ratios.

\begin{tabular}{|c|c|c|}
\hline $\begin{array}{l}\text { Bilateral concentric } \mathrm{H} / \mathrm{H} \text { ratios } \\
\mathrm{H} / \mathrm{H} \text { ratios }\end{array}$ & $\begin{array}{l}\text { Ipsilateral concentric } H / Q \text { ratios } \\
H / Q \text { ratios }\end{array}$ & $\begin{array}{l}\text { Mixed (eccentric/concentric) } \\
\text { H/Q ratios }\end{array}$ \\
\hline con $60 \mathrm{deg} / \mathrm{s}$ & con $60 \mathrm{deg} / \mathrm{s} \mathrm{R}$ and $\mathrm{L}$ & $60 \mathrm{deg} / \mathrm{s} \mathrm{R}$ and $\mathrm{L}$ \\
\hline con $120 \mathrm{deg} / \mathrm{s}$ & con $120 \mathrm{deg} / \mathrm{s} R$ and $\mathrm{L}$ & 120 deg/s $R$ and $L$ \\
\hline con $180 \mathrm{deg} / \mathrm{s}$ & con $180 \mathrm{deg} / \mathrm{s} R$ and $\mathrm{L}$ & $30 \mathrm{ecc} / 240 \mathrm{con} \mathrm{deg} / \mathrm{s} \mathrm{R}$ and $\mathrm{L}$ \\
\hline ecc $30 \mathrm{deg} / \mathrm{s}$ & con $240 \mathrm{deg} / \mathrm{s} R$ and $\mathrm{L}$ & \\
\hline \multicolumn{3}{|l|}{ ecc $120 \mathrm{deg} / \mathrm{s}$} \\
\hline ecc $60 \mathrm{deg} / \mathrm{s}$ & & \\
\hline
\end{tabular}

Abbreviations: con: concentric; ecc: eccentric; $R$ and L: right and left; H/Q: hamstring-to-quadriceps ratio.

The bilateral concentric strength ratios reported the knee flexors strength always by taking the weakest value compared with the strongest to obtain a value lower than 1. The conventional strength ratios were calculated for the same mode and speed of concentric muscle contraction. To avoid confusion, concentric muscle contraction corresponded to the muscle tension rises to meet the resistance then remains stable as the muscle shortens ${ }^{22}$. The mixed (concentric/eccentric) or functional ratios were established by reporting the eccentric performance of flexors and the concentric action of extensors ${ }^{8}$. Eccentric flexors contraction corresponded of the hamstring muscle lengthens as the resistance becomes greater than the force the muscle is producing ${ }^{23}$.

\section{Cut-offs used for prediction}

The cut-offs were fixed to 0.85 for bilateral ratios, to 0.47 for conventional ratios and to 0.80 for mixed ratios in reference to the study of Croisier et al. ${ }^{8}$

\section{Data analysis}

Two populations were identified according to the occurrence or not of hamstring injury during the soccer season which followed isokinetic strength tests. An ANOVA test was used to compare the mean of anthropometric parameters and various isokinetic strength ratios. For comparison of the ipsilateral strength ratios, the uninjured side of the population having presented hamstring injury was taken into account with both limbs of the population without hamstring injury. The results were considered significant at the $5 \%$ critical level $(p<0.05)$. Statistical analyses were carried out using SPSS 17.0 software (SPSS INC. Chicago, Illinois, USA).

The prediction of hamstring injury occurrence was assessed as the sensitivity (se), as the specificity (sp), and as the likelihood ratio for positive ( $L R+=s e / 1-s p)$ and negative (LR- = 1-se/sp) test providing the isokinetic strength ratios were independent using Spearman correlation analysis ${ }^{24}$. The sensitivity was the proportion of true positive that are correctly identified by the cut-offs used for prediction and the specificity was the proportion of true negative that are correctly identified by the same cut-offs ${ }^{25}$. A higher positive likelihood ratio corresponded to a higher differentiation power between the injured and uninjured leg. A likelihood ratio of more than 10 was strongly indicative of a new hamstring injury. A score of 5 to 10 was considered useful; 2 to 5 was moderately useful and less than 2 was considered unremarkable.

The prediction according to the severity of hamstring injury, moderate or major, was assessed by using the same statistical method after separation of the injured population according to the duration of the soccer stop lower or superior of 28 days ${ }^{15}$.

The prediction according to the period between isokinetic strength tests and hamstring injury was also analysed by the same statistical procedure after separation of three periods ( $\leq 1$ month or between 1 and 3 months or $>3$ months).

The calculation of the probability which the isokinetic parameter was present at the beginning of the soccer season before the hamstring injury occurrence was established from the hamstring injury prevalence for the studied population. It allowed to determine a pretest odds (prevalence/100-prevalence), then to calculate a post-test odds. The post-test probability in percentage was then established from the post-test odds. The estimated probability by taking into account several isokinetic ratios was calculated according to the same principle ${ }^{24}$.

\section{Results}

From 169 professional soccer players, who played at least 20 matches during one season, 17 players were excluded because they presented a lower limbs injury in the year which preceded isokinetic strength tests (5 knee surgeries, 1 patellar tendon injury and 11 quadriceps or hamstring injuries). Sixteen other players were excluded because they were not evaluated by isokinetic strength testing at the season beginning. Hundred and thirty six players thus realized 350 isokinetic strength tests during 10 consecutive seasons between 2001 and 2011 (Fig. 1). Eighty seven players were included at 2 occasions. Two hundred 


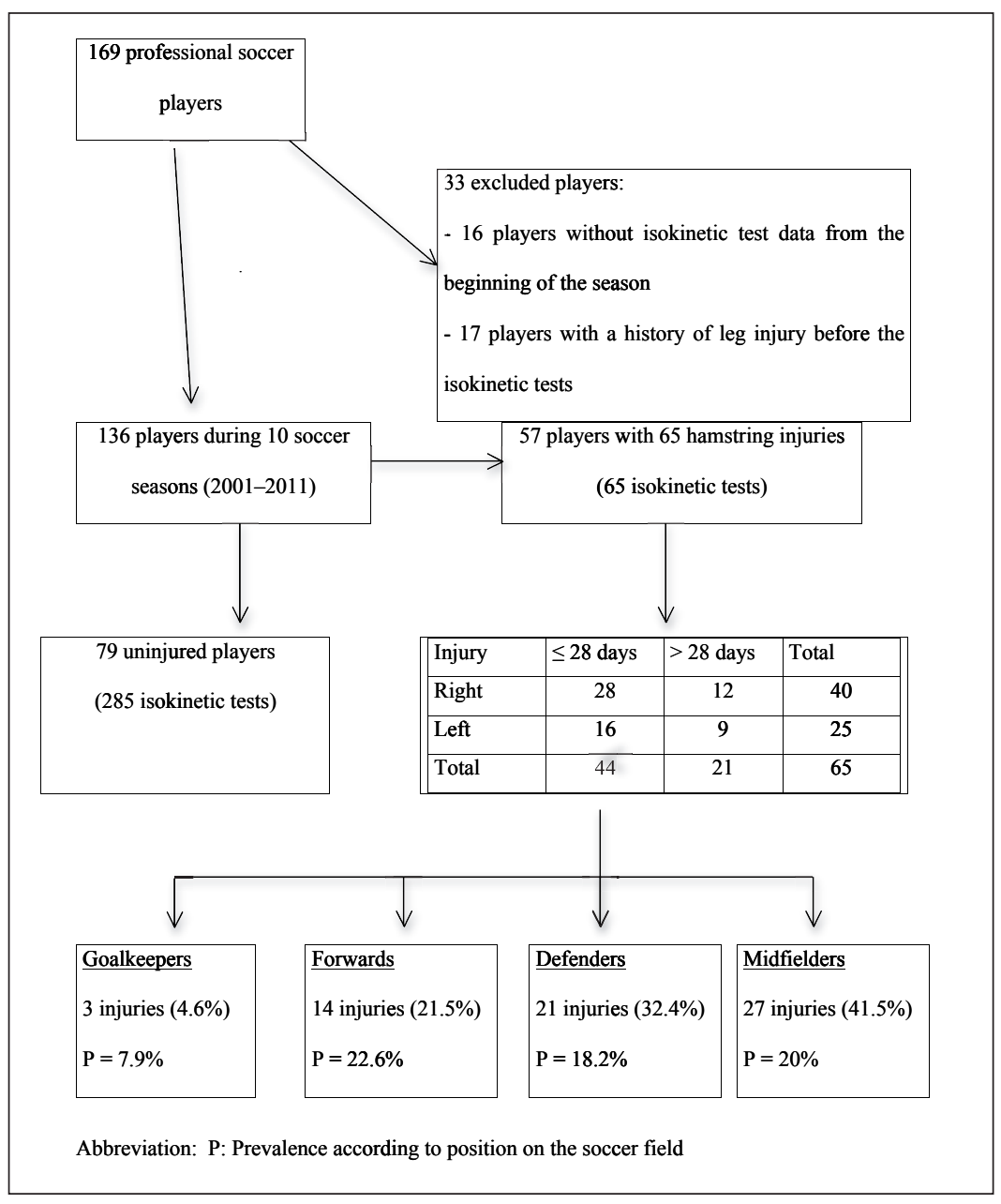

Figure 1: Study design.

eighty five isokinetic strength tests were carried out at 79 players who did not present hamstring injury. Fifty seven players presented 65 hamstring injuries during the season which followed isokinetic strength tests. Eight players presented injuries in both lower limbs. Forty injuries were diagnosed in right side against 25 in left. Hamstring injury prevalence for the studied population was thus of $18.6 \%$ (65/350). Twenty-one injuries were major (> 28 days) and 44 moderate ( $\leq$ 28 days). According to the playing position, 3 injuries were diagnosed at goalkeepers, 14 injuries at forwards, 21 injuries at defenders and 27 injuries at midfields.

The duration between isokinetic tests and hamstring injury was less than 1 month for 13 cases, between 1 and 3 months for 21 cases and upper to 3 months for 31 cases. No significant difference was found between both populations, injured and uninjured, for anthropometric parameters and various isokinetic strength ratios (Tabs. 3,4 ).

The prediction of hamstring injury occurrence was different according to the studied ratios (Tab. 5). Three ratios were useful with a positive diagnostic value upper to 2:

- The ipsilateral conventional concentric hamstring-
Table 3. Anthropometric parameters in injured and uninjured soccer players

\begin{tabular}{llll}
\hline & Uninjured & Injured & p \\
\hline Age (Years) & $22.5 \pm 4.8$ & $25.2 \pm 4.2$ & ns \\
Weight $(\mathrm{kg})$ & $75.2 \pm 6.9$ & $76.1 \pm 6$ & ns \\
Height $(\mathrm{cm})$ & $180.4 \pm 5.8$ & $180.8 \pm 4.6$ & ns \\
BMI (kg/cm²) & $23.1 \pm 1.5$ & $23.3 \pm 1.2$ & ns \\
\hline
\end{tabular}

Abbreviations: BMI: body mass index; ns: non-significant.

to-quadriceps $(\mathrm{H} / \mathrm{Q})$ ratio at $180 \mathrm{deg} / \mathrm{s}$ lower than 0.47 shown a sensitivity of $7 \%$, a specificity of $97 \%$ and a prediction probability of $36.9 \%$.

- The ipsilateral mixed 30 eccentric/240 concentric hamstring-to-quadriceps ratio lower than 0.8 shown a sensitivity of $2.5 \%$, a specificity of $99 \%$ and a prediction probability of $40.1 \%$.

- The bilateral concentric hamstring-to-hamstring $(\mathrm{H} / \mathrm{H})$ ratio at $60 \mathrm{deg} / \mathrm{s}$ lower than 0.85 shown a sensitivity of $32 \%$, a specificity of $82 \%$ and a prediction probability of $34 \%$. 
Table 4. Isokinetic strength ratios in injured and uninjured soccer players.

\begin{tabular}{|c|c|c|c|c|}
\hline Number of Players & $H / Q$ ratios & Uninjured (U) & Injured (I) & $\mathbf{p}$ \\
\hline $684(\mathrm{I}=64, \mathrm{U}=620)$ & con $60 \mathrm{deg} / \mathrm{s}$ & $0.66 \pm 0.10$ & $0.66 \pm 0.11$ & 0.68 (ns) \\
\hline $402(\mathrm{I}=38, \mathrm{U}=364)$ & con $120 \mathrm{deg} / \mathrm{s}$ & $0.65 \pm 0.17$ & $0.68 \pm 0.16$ & 0.28 (ns) \\
\hline $322(\mathrm{I}=26, \mathrm{U}=296)^{*}$ & con $180 \mathrm{deg} / \mathrm{s}$ & $0.71 \pm 0.21$ & $0.70 \pm 0.22$ & 0.94 (ns) \\
\hline \multirow[t]{2}{*}{$518(\mathrm{I}=49, \mathrm{U}=469)$} & con $240 \mathrm{deg} / \mathrm{s}$ & $0.84 \pm 0.44$ & $0.89 \pm 0.61$ & $0.50(\mathrm{~ns})$ \\
\hline & Mixed ratios & & & \\
\hline $136(I=10, U=126)^{*}$ & ecc/con $60 \mathrm{deg} / \mathrm{s}$ & $0.88 \pm 0.20$ & $0.83 \pm 0.24$ & $0.53(\mathrm{~ns})$ \\
\hline $206(\mathrm{I}=20, \mathrm{U}=186)^{*}$ & ecc/con $120 \mathrm{deg} / \mathrm{s}$ & $1.51 \pm 0.26$ & $1.46 \pm 0.48$ & 0.93 (ns) \\
\hline \multirow[t]{2}{*}{$392(\mathrm{I}=39, \mathrm{U}=353)$} & $30 \mathrm{ecc} / 240 \mathrm{con} \mathrm{deg} / \mathrm{s}$ & $1.32 \pm 0.26$ & $1.31 \pm 0.24$ & $0.72(\mathrm{~ns})$ \\
\hline & $\mathrm{H} / \mathrm{H}$ ratios & & & \\
\hline $343(\mathrm{I}=64, \mathrm{U}=279)$ & con $60 \mathrm{deg} / \mathrm{s}$ & $0.89 \pm 0.09$ & $0.88 \pm 0.10$ & 0.29 (ns) \\
\hline $281(\mathrm{I}=53, \mathrm{U}=228)$ & con $120 \mathrm{deg} / \mathrm{s}$ & $0.74 \pm 0.20$ & $0.74 \pm 0.16$ & 0.95 (ns) \\
\hline $160(\mathrm{I}=26, \mathrm{U}=134)^{*}$ & con $180 \mathrm{deg} / \mathrm{s}$ & $0.87 \pm 0.12$ & $0.89 \pm 0.12$ & 0.64 (ns) \\
\hline $198(\mathrm{I}=41, \mathrm{U}=157)$ & ecc $30 \mathrm{deg} / \mathrm{s}$ & $0.89 \pm 0.07$ & $0.87 \pm 0.10$ & 0.65 (ns) \\
\hline $67(\mathrm{I}=10, \mathrm{U}=57)^{*}$ & ecc $60 \mathrm{deg} / \mathrm{s}$ & $0.85 \pm 0.11$ & $0.81 \pm 0.11$ & $0.57(\mathrm{~ns})$ \\
\hline $188(\mathrm{I}=35, \mathrm{U}=153)$ & ecc $120 \mathrm{deg} / \mathrm{s}$ & $0.87 \pm 0.11$ & $0.86 \pm 0.10$ & $0.72(\mathrm{~ns})$ \\
\hline
\end{tabular}

Abbreviations: con: concentric; ecc: eccentric; ns: non-significant; I: injured leg; U: uninjured leg.

Table 5. Ability of isokinetic strength ratios to predict hamstring injury.

\begin{tabular}{|c|c|c|c|c|c|c|c|}
\hline & Se $(\%)$ & $\mathrm{Sp}(\%)$ & PPV (\%) & PNV (\%) & LR+ & LR- & P signe+ \\
\hline \multicolumn{8}{|c|}{$H / Q$ ratios (cut-off $<0.47$ ) } \\
\hline con $60 \mathrm{deg} / \mathrm{s}$ & 3.1 & 98.2 & 15.4 & 91 & 1.74 & 0.98 & 28.6 \\
\hline con $120 \mathrm{deg} / \mathrm{s}$ & 5.2 & 89.3 & 4.8 & 90 & 0.49 & 1.06 & 10 \\
\hline con $180 \mathrm{deg} / \mathrm{s}$ & 7.7 & 97 & 18 & 92.3 & $2.56^{*}$ & 0.95 & $36.8^{*}$ \\
\hline con $240 \mathrm{deg} / \mathrm{s}$ & 0 & 99.8 & 0 & 91 & 0 & 1 & 0 \\
\hline \multicolumn{8}{|c|}{ Mixed ratios (cut-off $<0.8$ ) } \\
\hline ecc/con $60 \mathrm{deg} / \mathrm{s}$ & 30 & 65 & 63.8 & 92 & 0.86 & 1.08 & 16.3 \\
\hline ecc/con $120 \mathrm{deg} / \mathrm{s}$ & 5 & 96 & 12.5 & 90.5 & 1.25 & 0.99 & 22.1 \\
\hline $30 \mathrm{ecc} / 240 \mathrm{con} \mathrm{deg} / \mathrm{s}$ & 2.5 & 99.1 & 25 & 90.2 & $2.94^{*}$ & 0.98 & $40.1^{*}$ \\
\hline \multicolumn{8}{|c|}{$\mathrm{H} / \mathrm{H}$ ratios (cut-off $<0.85$ ) } \\
\hline con $60 \mathrm{deg} / \mathrm{s}$ & 32.8 & 85.4 & 22.5 & 85.4 & $2.26^{*}$ & 0.78 & $34^{*}$ \\
\hline con $120 \mathrm{deg} / \mathrm{s}$ & 64.1 & 33.7 & 18.3 & 80.2 & 0.96 & 1.06 & 17.9 \\
\hline con $180 \mathrm{deg} / \mathrm{s}$ & 23 & 73.8 & 14.6 & 83.1 & 0.88 & 1.04 & 16.7 \\
\hline ecc $30 \mathrm{deg} / \mathrm{s}$ & 29.2 & 72.6 & 21.8 & 79.7 & 1.06 & 0.97 & 19.6 \\
\hline ecc $60 \mathrm{deg} / \mathrm{s}$ & 60 & 59.5 & 20.6 & 89.4 & 1.48 & 0.98 & 25.2 \\
\hline ecc $120 \mathrm{deg} / \mathrm{s}$ & 42.8 & 72.5 & 26.3 & 84.7 & 1.48 & 0.98 & 25.2 \\
\hline
\end{tabular}

Abbreviations: con: concentric; ecc: eccentric; Se: sensitivity; Sp: specificity; PPV and PNV: positive and negative predictive values; LR: likelihood ratio; P signe+: positive probability of hamstring injury if the ratio is less than the cut-off value *Useful parameter for prediction 
When these ratios were associated, the prediction became major (accumulated LR+: 17.1) and the probability reached $79.4 \%$ ).

In practice, 22 injuries of 65 have been able to be predicted by the bilateral 60 concentric $\mathrm{H} / \mathrm{H}$ ratio < 0.85 because of the sensitivity of $32 \%$. The association of the 3 aforesaid ratios would have predicted 7 injuries of more (29/65) according to the studied population. According to injury severity, the most useful prediction was brought by the 60 concentric $\mathrm{H} / \mathrm{Q}$ ratio $<0.47$ with a probability of $24.4 \%$ for the moderate injuries and of $23 \%$ for the major injuries. This ratio was also useful for the prediction according to the period of the occurrence of a new hamstring injury. The prediction probability was of $49.3 \%$ for the 1 month period (LR+: 4.27 ), of $54.6 \%$ for the 1 to 3 months period (LR+: 5.27$)$ and more limited beyond 3 months (29.5\%; LR+: 1.84).

\section{Discussion}

No anthropometric difference was highlighted between the subjects who presented or not hamstring injury during the season which followed isokinetic strength tests. Isokinetic strength ratios were not different between the two populations. These results are comparable to those of the literature about soccer and Australian soccer players or other sports prospectively studied $1,3,10$. According to individual playing positions, the hamstring injury prevalence was higher at forwards $(22.6 \%)$ then at midfields $(20 \%)$, at defenders (18.2\%) and finally at goalkeepers (7.9\%). It could be linked with different muscle strength profile. Besides, muscle injuries at soccer player would occur more during competition than during training. We were not able to study this parameter because it was not enough listed.

Some isokinetic strength ratios are useful for hamstring injury prediction during the sports season. The ipsilateral mixed 30 eccentric/240 concentric H/Q ratio was the most predictive followed by the conventional 180 concentric $H / Q$ ratio and the bilateral 60 concentric $\mathrm{H} / \mathrm{H}$ ratio. Furthermore, the association of these 3 ratios was useful to identify hamstring injury occurring during the season following isokinetic strength tests (probability of $79 \%$ ). Twenty two injuries on 65 have been able to be predicted by the bilateral 60 concentric $\mathrm{H} / \mathrm{H}$ ratio $<0.85$. The association of 3 ratios would have allowed the prediction of 7 additional injuries (29/65) limited by the sensitivity defect of the strength ratios. The bilateral 60 concentric $\mathrm{H} / \mathrm{H}$ ratio presented a sensitivity of $32 \%$ in comparison with the $3 \%$ sensitivity of the 2 other ratios. The defect of sensitivity is explained by the used cut-off values which vary from a study to the other one. It explains finally the contradictory results of the literature. When the cut-off of 0.6 is chosen instead of 0.47 for the conventional concentric $\mathrm{H} / \mathrm{Q}$ ratios, no possibility of prediction was highlighted $3,10,14$. According to a prospective method, Dauty et al. had identified only a single player on 5 who was going to injure ${ }^{10}$. In fact, low ipsilateral or asymmetric bilateral strength ratios would be rather the consequence of hamstring injury as shown by the prediction of a previous injury and not a new injury ${ }^{3,10}$. At the American football player, the conventional 60 concentric $H / Q$ ratio $<0.6$ is not predictive being shown a sensitivity of $51 \%$ and a specificity of $52 \%{ }^{14}$. Also, a $10 \%$ asymmetry according to the bilateral 60 concentric $\mathrm{H} / \mathrm{H}$ ratio was shown at $41 \%$ of injured players but also at $43 \%$ of uninjured players ${ }^{14}$. This 0.9 cut-off, superior than the one used of 0.85 in this study, was able to be at the origin of a specificity defect which would have improve the prediction because of the positive likelihood ratio calculation (Se:1-Sp).

On the contrary, a prediction was possible by using or a canonical coefficient correlation or a linear regression with the conventional 60 concentric $H / Q$ ratio lower than $0.6^{26,27}$. The risk of hamstring injury occurrence would be multiplied by seventeen at the shortdistance runners according to Yeung et al. ${ }^{27}$. However, these results are debatable because of the ratios calculation which reported the injured side on the healthy side knowing that there was a significant difference between the injured and uninjured populations. This method of ratios calculation was able to be at the origin of bias because it amounts to know the injured players before the isokinetic strength measurements. Furthermore, the weak number of included subjects could explain a lack of power of results. Only 6 injured subjects on 37 studied sportsmen were reported 26 .

On the contrary, Croisier et al. showed predictive strength ratios by using lower cut-off values ${ }^{8}$. That is why our study used the same cut-off values. It was noticed that the professional soccer players who were victims of hamstring injury presented more frequently a pre-existing muscle imbalance ${ }^{28}$. From 77 professional soccer players recruited in 4 different teams (Brazil, Belgium and France), players were injured five times more in case of muscle imbalance in 9 months following isokinetic strength tests. However, $47 \%$ of injured players were also unbalanced for two calculated different strength ratios at least ${ }^{8}$. The mixed 30 eccentric/240 concentric $H / Q$ ratio represented the strength ratio which most identified unbalanced players (87\%: 187 on 216 studied cases) ${ }^{8}$. So, by bringing together injured players during the following season, the risk of hamstring injury occurrence was either multiplied by 5 but by 4 (16 against $4 \%$ ) in case of imbalance objectified by the mixed ratio lower than 0.8. By taking back the results of this study, the calculation of the risk of injury occurrence is particular. If it is added all injured players who presented a pre-existing muscle imbalance, the risk of injury becomes then lower than three (11 against $4 \%$ ) and if the number of lost subjects is considered, the prevalence of injury becomes very weak $(7.5 \%$ : 35 injuries for 462 included players). Only injuries responsible for more four weeks of sports stop had been considered. By taking into account all these remarks, the conclusions of this study would have been more qualified ${ }^{8}$. 
In spite of the limits concerning the mixed 30 eccentric/240 concentric $H / Q$ ratio, this one is useful for the prediction of hamstring injury if the cut-off value of 0.8 is chosen. The interest of this strength ratio would be to consider the knee flexors eccentric strength which mimes the muscle injury mechanism. The fact of reporting the flexors eccentric strength to the extensors concentric strength at $240 \mathrm{deg} / \mathrm{s}$ isokinetic fast speed, would feign the hamstring braking during a quickly contraction of the quadriceps ${ }^{19}$. However, our results are to be qualified being admitted a multifactorial origin of hamstring injury6,29,30,31. The cut-off of 0.8 chosen raises question. Our study showed means of 1.31 and 1.32 for the injured and the uninjured population respectively. By comparison, Lehance et al. showed same means of 1.40 at the professional soccer player ${ }^{1}$. With a cut-off of 0.8 , the specificity is thus very high (99\%) but the sensitivity is very low $(2.5 \%)$. So, few players are really identified as risk of injury. On the other hand, when a player is identified, the prediction of injury is very high. Only 2 injured players on 65 presented such strength ratio.

The bilateral 60 concentric $\mathrm{H} / \mathrm{H}$ ratio presented in fact most interest (Probability of $34 \%$ ). Used only, one lesion of $3(22 / 65)$ can be predicted because of the sensibility of $32 \%$ while the specificity is correct $(85 \%)$. From a practical point of view, this ratio is thus more interesting that the mixed ratio previously describes. However, Dauty et al., had prospectively shown that 2 of 5 new injured subjects presented an asymmetry of more than $15 \%$, but on the contrary in favour of the injured side ${ }^{10}$. This asymmetry of more than $15 \%$ is thus interesting to know while the strongest limb can also be at risk of injury maybe by compensation.

The conventional 60 concentric $H / Q$ ratio presented a real interest close to the conventional ratio calculated at $180 \mathrm{deg} / \mathrm{s}$. This strength ratio allows especially a prediction for the severity of hamstring injury (probability of $25 \%$ ) and whatever the period between the occurrence of the injury and the isokinetic strength measurement. The probability reached almost 50\% until 3 months after isokinetic strength tests and decreased in $30 \%$ after 3 months. To our knowledge, no prediction was established for these two parameters, nevertheless important to consider. Croisier et al., had only studied the major injuries of a more than 30 days duration and a prediction seems more complex to establish when a muscle injury occurs at distance of isokinetic strength tests ${ }^{8}$. To confirm this prediction, it will nevertheless be necessary to study a largest number of soccer players.

From three aforesaid strength ratios, the prediction was looked by associating them. The probability of the occurrence of hamstring injury reaches then almost $80 \%$ if a professional soccer player present a mixed 30 eccentric/240 concentric $H / Q$ ratio lower than 0.8 , a conventional 180 concentric $H / Q$ ratio lower than 0.47 and a bilateral 60 concentric $\mathrm{H} / \mathrm{H}$ ratio lower than 0.85 . This statistical method countered attractive because of these three different calculated parameters are independent from each other. However, none of the studied players presented such a strength profile. The association of these 3 ratios allowed identifying only 7 additional subjects by comparison with the use of the bilateral 60 concentric $\mathrm{H} / \mathrm{H}$ ratio in an isolated way.

Other statistical limits were also present. The prevalence of muscular injuries for the studied population intervened for the calculation of the posteriori probability of diagnostic. This one was of $18.6 \%$ on ten seasons and confirmed the high frequecy of hamstring injuries at professional soccer players. A comparable between 11 in $27 \%$ incidence, was described at soccer players, at Australian or American football, even if these last two sports are not exactly comparable ${ }^{3,10,14,26}$. However, the prevalence value did not influence the calculation of the likelihood ratio, so that the choice of the predictive ratios remained valid 24 .

Otherwise, the fact that three isokinetic protocols were used during 10 seasons was able to represent a bias for obtaining maximal isokinetic peak torque. It gave some historical explanation by the fact that in 2001, the eccentric isokinetic measurements were rare at the professional soccer player's and that the mixed (eccentric/concentric) ratio, used by Croisier et al., had not been published yet ${ }^{12}$. However, the various protocols always associated two concentric followed by two eccentric series with a comparable number of repetitions, but according to different angular speeds. This bias connected to the use of different protocol was weak thus doubtless. On the other hand, the different protocols allowed estimating the interest of 13 different ratios by using different angular speeds.

\section{Perspective}

According to the cut-off values used, the prediction of hamstring injury occurrence is possible from isokinetic strength measurement at the beginning of season. Certain strength ratios are useful in practice to detect a third of soccer players who are going to injure in the season. Statistically, the association of the 30 eccentric/240 concentric $H / Q, 60$ concentric $H / Q$ and 60 concentric $\mathrm{H} / \mathrm{H}$ ratios would allow a probability of $79 \%$ according to the cut-off values of $0.8 ; 0.47$ and 0.85 respectively. From these results, the calculation of the various aforesaid strength ratios can be made according to the isokinetic protocol at the beginning of following season:

- 3 concentric sets at $60 \mathrm{deg} / \mathrm{s}$ for knee extensors and flexors

- 5 concentric sets at $180 \mathrm{deg} / \mathrm{s}$ for knee extensors and flexors

- 5 concentric sets at $240 \mathrm{deg} / \mathrm{s}$ for knee extensors and flexors

- 5 eccentric sets at $30 \mathrm{deg} / \mathrm{s}$ only for knee flexors.

\section{Conflict of interests}

The Authors declare that they have no conflict of interests regarding the publication of this paper. 


\section{References}

1. Lehance C, Binet J, Bury T, Croisier JL. Muscular strength, functional performances and injury risk in professional and junior elite soccer players. Scand J Med Sci Sports. 2009; 19:243-251.

2. Dolman B, Verrall G, Reid I. Physical principles demonstrate that the biceps femoris muscle relative to the other hamstring muscles exerts the most force: implications for hamstring muscle strain injuries. Muscles Ligaments Tendons J. 2014;4:371377.

3. Bennell K, Wajswelner H, Lew P, Schall-Riaucour A, Leslie S, Plant $\mathrm{D}$, Cirone J. Isokinetic strength testing does not predict hamstring injury in Australian Rules footballers. Br J Sports Med. 1998;32:309-314.

4. Maffulli N, Oliva F, Frizziero A, et al. ISMuLT Guidelines for muscle injuries. Muscles Ligaments Tendons J. 2014;3:241249.

5. Guillodo Y, Here-Dorignac C, Thoribé B, Madouas G, Dauty $M$, Tassery F, Saraux A. Clinical predictors of time to return to competition following hamstring injuries. Muscles Ligaments Tendons J. 2014;4:386-390.

6. Orchard $\mathrm{J}$. Is there a relationship between ground and climatic conditions and injuries in football? Sports Med. 2002;32:419432.

7. Schmitt B, Tim T, McHugh M. Hamstring injury rehabilitation and prevention of reinjury using lengthened state eccentric training: a new concept. Int J Sports Phys Ther. 2012;7:333341.

8. Croisier J-L, Ganteaume S, Binet J, Genty M, Ferret JM. Strength imbalances and prevention of hamstring injury in professional soccer players. Am J Sports Med. 2008;36:14691475.

9. Croisier J-L and Crielaard J-M. Hamstring muscle tear with recurrent complaints: an isokinetic profile. Isokinet Exerc Sci. 2000;8:175-180.

10. Dauty M, Potiron-Josse M, Rochcongar P. Consequences and prediction of hamstring muscle injury with concentric and eccentric isokinetic parameters in elite soccer players. Ann Readapt Med Phys. 2003;46:601-606.

11. Askling C, Karlsson J, Thorstensson A. Hamstring injury occurrence in elite soccer players after preseason strength training with eccentric overload. Scand J Med Sci Sports. 2003;13: 244-250.

12. Croisier $\mathrm{J}-\mathrm{L}$, Forthomme $\mathrm{B}$, Namurois $\mathrm{M}-\mathrm{H}$, Vanderthommen $\mathrm{M}$, Crielaard J-M. Hamstring muscle strain recurrence and strength performance disorders. Am J Sports Med. 2002;30: 199-203.

13. Proske $U$, Morgan DL, Brockett CL, Percival P. Identifying athletes at risk of hamstring strains and how to protect them. Clin Exp Pharmacol Physiol. 2004;31:546-550.

14. Zvizac JE, Toriscelli TA, Merrick S, Kiebzak GM. Isokinetic concentric quadriceps and hamstring strength variables from the NFL scouting combine are not predictive of hamstring injury in the first-year professional football players. Am J Sports Med. 2013;41:1511-1518.

15. Hagglund M, Walden M, Bahr R, Ekstrand J. Methods for epidemiological study of injuries to professional football players: developing the UEFA model. Br J Sports Med. 2005;39:340346.

16. Padulo J, Olivia F, Frizziero A, Maffulli N. Muscles, Ligaments and tendons Journal. Basic principles and recommendations in clinical and field science research. MLTJ. 2013;3(4):250-252.

17. Dauty $M$, Rochcongar $P$. Isokinetic and anterior cruciate ligament reconstruction with hamstrings or patella tendon graft: Analysis of literature. Int J Sports Med. 2005;26:599-606.

18. Junge A, Dvorak J, Graf-Bauman T, Peterson L. Football injuries during FIFA tournaments and the Olympic games 19982001. Development and implementation of an Injury-Reporting System. Am J Sports Med. 2004;32(Suppl 1):80S-89S.

19. Aagaard P, Simonsen EB, Trolle M, Bangsbo J, Klausen K. Isokinetic hamstring/quadriceps strength ratio: influence from joint angular velocity, gravity correction and contraction mode. Acta Physiol Scand. 1995;154:421-427.

20. Ayala F, De St Croix M, de Baranda S, Santonja F. Absolute reliability of hamstring to quadriceps strength imbalance ratios calculated using peak torque, joint angle-specific torque and joint ROM-specific torque values. Int J Sports Med. 2012;33: 909-916.

21. Impellizzeri FM, Bizzini M, Rampinini E, Cereda F, Maffiuletti NA. Reliability of isokinetic strength imbalance ratios measured using the Cybex NORM dynamometer. Clin Physiol Funct Imaging. 2008;28:113-119.

22. Padulo J, Chamari K, Concu A, Dal Pupo J, Laffaye G, Moura Zagatto A, Ardigo LP. Concentric and eccentric: muscle contraction or exercise? New perspective. Muscles Ligaments Tendons J. 2014;4:158.

23. Padulo J, Laffaye G, Ardigo LP, Chamari K. Concentric and eccentric: muscle contraction or exercise? J Hum Kinet. 2013;37:5-6

24. Jaeschle R, Guyatt GH, Sackett DL. For the evidence-based medicine working group. User's guides to the medical literature. III. How to use an article about a diagnosis test. B. What are the results and will they help me in caring for my patients? JAMA. 1994;271:703-707.

25. Altman DG, Bland JM. Diagnosis test 1: sensitivity and specificity. BMJ. 1994;308:1552.

26. Orchard J, Marsden J, Lord S, Garlick D. Preseason hamstring muscle weakness associated with hamstring muscle injury in Australian footballers. Am J Sports Med. 1997;25:81-85.

27. Yeung SS, Suen AMY, Yeung EW. A prospective cohort study of hamstring injuries in competitive sprinters: preseason muscle imbalance as a possible risk factor. $\mathrm{Br} J$ Sports Med. 2009; 43:589-594.

28. Croisier J-L, Reveillon V, Ferret JM, Cotte T, Genty M, Popovich N. Isokinetic assessment of knee flexors and extensors in professional soccer players. Isokinet Exerc Sci. 2003;11:61-62.

29. Croisier J-L. Factors associated with recurrent hamstring injuries. Sports Med. 2004;34:68-95.

30. Gabbe BJ, Bennell K, Finch CF, Wajswelner H, Orchard JW Predictors of hamstring injury at the elite level of Australian football. Br J Sport Med. 2006;16:7-13.

31. Small K, McNaughton L, Greig M, Lovell R. The effects of multidirectional soccer-specific fatigue on markers of hamstring injury risk. J Sci Med Sport. 2010;13:120-125. 